Trapianti e Terapie Cellulari “C. Melzi”, DAME, ASUI, Udine, Italy;

${ }^{12}$ Department of Oncology and Hematology, Fondazione Istituto Nazionale dei Tumori Milano University of Milano, Milano, Italy;

${ }^{13}$ Department of Molecular Medicine, University of Pavia, Division of Hematology, Fondazione IRCCS Policlinico S. Matteo, Pavia, Italy;

${ }^{14}$ Medical Physics Unit, Azienda Ospedaliera S. Croce e Carle, Cuneo, Italy; ${ }^{15}$ Hematology Unit, Department of Translational and Precision Medicine, Sapienza University of Rome, Roma, Italy; ${ }^{16}$ Division of Hematology, ASST Grande ospedale Metropolitano Niguarda, Milano, Italy; ${ }^{17}$ Department of Hematology and Developmental Therapeutics, Istituto Nazionale Tumori, Fondazione 'G. Pascale' IRCCS, Napoli, Italy; ${ }^{18}$ UO Ematologia, OC Rimini AUSL Romagna, Rimini, Italy;

${ }^{19}$ Hematology, University Hospital "Ospedale di Circolo e Fondazione Macchi" - ASST Sette Laghi, University of Insubria, Varese, Italy;

${ }^{20}$ Ematologia, Università degli Studi di Firenze, Firenze, Italy;

${ }^{21}$ Hematology, AOU Maggiore della Carità, Novara, Italy; ${ }^{22}$ Hematolgy Unit, ASST-Monza, Monza, Italy; ${ }^{23}$ Clinical and Experimental OncoHaematology Unit, Centro di Riferimento Oncologico, IRCCS, Aviano, Italy; ${ }^{24}$ Divisione di Diagnosi Ematopatologica, Istituto Europeo di Oncologia, Milano, Italy; ${ }^{25}$ S.C. Ematologia, Arcispedale S. Maria NuovaIRCCS, Reggio Emilia, Italy

Introduction: Rituximab maintenance after frontline R+chemo prolongs remissions and is a widely adopted treatment option for patients with follicular lymphoma (FL). Moreover, metabolic and molecular response assessed with FDG-PET and MRD have been confirmed as strong predictors of patients' survival thus suggesting the benefit of a response adapted maintenance strategy as post induction therapy. Methods: FOLL12 is a multicenter, randomized, phase III, non inferiority study comparing standard vs response adapted maintenance in patients with stage II-IV, previously untreated, intermediate-high risk FL according to FLIPI2, requiring therapeutic intervention. All patients received induction immunochemotherapy (ICT) with 6 cycles of RCHOP or 6 cycles of R-bendamustine both followed by 2 additional doses of rituximab. After induction ICT, patients in the standard arm were treated with bimonthly rituximab doses for up to two years. Patients in the experimental arm were managed according to centrally reviewed metabolic and molecular response (i.e. Complete metabolic and molecular response: no therapy; Complete metabolic response without molecular response: 4 weekly rituximab doses; Lack of metabolic response (Deauville score 4-5): radiommunotherapy with ibritumomab tiuxetan followed by standard rituximab maintenance). Primary study endpoint was 3 years progression free survival (PFS) by intention to treat. A sample size of 770 evaluable patients was planned assuming a reference of $3 y$ PFS at $70 \%$ with a non inferiority margin of $7 \%$ between arms.

Results: A total of 790 eligible patients were randomized to standard (394 patients) or experimental (396 patients) arm. Groups were well balanced according to patient characteristics and response rates. At the end of induction therapy $88 \%$ of cases resulted PET- and $91 \%$ MRD-. After a median follow-up of 37 months (range 1-71), the 3 -year overall survival (OS) and PFS were $96 \%$ and $76 \%$, respectively.
An interim analysis, planned at the occurrence of $70 \%$ events, was anticipated at the occurrence of $50 \%$ of events and submitted to the external data safety monitoring committee (DSMC). The analysis showed that response oriented experimental arm resulted significantly inferior to the standard maintenance arm in terms of PFS (estimated 3-year PFS, $68 \%$ vs. 84\%; hazard ratio for PFS, $2.05[95 \% \mathrm{Cl}$ 1.50-2.81; $\mathrm{P}<0.0001]$ ). The DSMC considered unlikely that longer follow-up could modify the results and was favorable in publishing these data.

Conclusions: In patients with intermediate-high risk FL according to FLIPI2 and requiring systemic therapy, omission of R-maintenance resulted in a significantly lower 3-year PFS, despite the attainment of a post-induction complete metabolic response.

Keywords: follicular lymphoma (FL); minimal residual disease (MRD); positron emission tomography (PET).

Disclosures: Federico, M: Honoraria: Janssen, Gilead, Medlmmune. Mannina, D: Consultant Advisory Role: Janssen, AbbVie. Ferrero, S: Consultant Advisory Role: Janssen; Honoraria: Janssen, Gilead, Servier. Galimberti, S: Other Remuneration: Speaker for Roche, Takeda, Celgene, Janssen. Cavallo, F: Consultant Advisory Role: Takeda, Janssen. Arcaini, L: Consultant Advisory Role: CELGENE, SANDOZ, Janssen-Cilag, VERASTEM; Research Funding: Gilead. Chauvie, S: Honoraria: IAEA, CONSIP and Sirtex; Research Funding: Roche, Takeda, Fondazione Cassa di Risparmio di Cuneo and AIRC; Other Remuneration: Travel/accommodation from GE Healthcare, Philips Medical System, Sirtex, BTG, Terumo. He is co-founder of Dixit srl, spin-off of University of Torino and INFN. Rusconi, C: Consultant Advisory Role: Roche. Pinto, A: Consultant Advisory Role: Servier, Roche, Merck Sharp \& Dohme; Honoraria: Roche/Genetech, Merck Sharp \& Dohme, Bristol Myers Squibb, Celgene, Servier; Other Remuneration: EDO-Mundipharma (patenting process). Kovalchuk, S: Other Remuneration: Roche. Nassi, L: Consultant Advisory Role: Takeda, Janssen, MSD. Luminari, S: Consultant Advisory Role: Roche, Celgene, Sandoz, Gilead.

\section{5 \\ INTERIM UPDATE FROM A PHASE 2 MULTICENTER STUDY OF TAZEMETOSTAT, AN EZH2 INHIBITOR, IN PATIENTS WITH RELAPSED OR REFRACTORY FOLLICULAR LYMPHOMA}

F. Morschhauser $^{1}$ | H. Tilly ${ }^{2}$ | A. Chaidos ${ }^{3}$

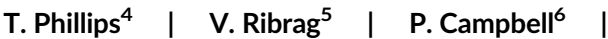

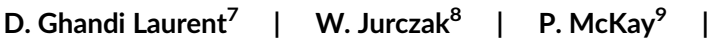

S. Opat ${ }^{10}$ | J. Radford ${ }^{11}$ | A. Rajarethinam ${ }^{12}$ |

J. Yang $^{13}$ | H. Howell ${ }^{14}$ | K.J. Newberry ${ }^{15}$ |

D. Adib $^{16}$ | G. Salles ${ }^{17}$

${ }^{1}$ Hematology-Transfusion, Centre Hospitalier Universitaire, Lille, France; ${ }^{2}$ Hematology, Centre de Lutte Contre le Cancer Henri Becquerel, Rouen, France; ${ }^{3}$ Medicine, Centre for Haematology, Department of Medicine, Imperial College London, Imperial College Healthcare NHS Trust, 
Hammersmith Hospital, London, United Kingdom; ${ }^{4}$ Hematology and Oncology, University of Michigan, Ann Arbor, United States; ${ }^{5}$ DITEP, Gustave Roussy, Villejuif, France; ${ }^{6}$ Hematology, Barwon Health, Geelong, Australia; ${ }^{7}$ Hematology, Hematology Institute University Hospital School of Medicine, Caen, France; ${ }^{8}$ Hematology, UJCM, Kraków, Poland; ${ }^{9}$ Hematology, Beatson West of Scotland Cancer Centre, Glasgow, United Kingdom; ${ }^{10}$ Hematology, Monash University, Clayton, Australia; ${ }^{11}$ Medical Oncology, University of Manchester and the Christie NHS Foundation Trust, Manchester Academic Health Science Centre, Manchester, United Kingdom; ${ }^{12}$ Data Management, Epizyme, Cambridge, United States; ${ }^{13}$ Biostatistics, Epizyme, Cambridge, United States; ${ }^{14}$ Clinical Operations, Epizyme, Cambridge, United States; ${ }^{15}$ Clinical Research, Epizyme, Cambridge, United States; ${ }^{16}$ Clinical Development, Epizyme, Cambridge, United States; ${ }^{17}$ Hematology, Lyon-Sud Hospital Centre, Pierre-Bénite, France

Introduction: Relapsed or refractory (R/R) follicular lymphoma $(\mathrm{FL})$ remains an area of unmet medical need and treatments with novel mechanisms of action are desirable. The histone methyltransferase $\mathrm{EZH} 2$ is an important regulator of the germinal center (GC) reaction that is involved in preventing terminal differentiation of GC B-cells. EZH2 activating mutations are present in $\sim 20 \%$ of $F L$ patients (pts) and are postulated to be oncogenic drivers. Tazemetostat, a selective, oral EZH2 inhibitor has shown antitumor activity in a phase $1 / 2$ study that included non-Hodgkin lymphoma pts with mutant (MT) or wildtype (WT) EZH2tumors, providing rationale for further investigation. Methods: This open-label, multicenter, phase 2 study of tazemetostat enrolled pts with either MT or WT EZH2 R/R diffuse large B-cell lymphoma or FL (Grade 1-3b). Key inclusion criteria included: age $\geq 18$ years, $\geq 2$ prior treatment regimens, and measurable disease. Tumor tissue was analyzed for EZH2 hot spot activating mutations (Y646X, A682G, A692V) using a cobas ${ }^{\circledR}$ EZH2 Mutation Test (Roche Molecular Systems, investigational use only). Tazemetostat $800 \mathrm{mg}$ was administered orally, twice daily. Response was assessed every 8 weeks using 2007 IWG-NHL criteria. The primary endpoint was ORR (CR + PR). Secondary endpoints included PFS and safety/tolerability. Efficacy and safety were analyzed for the EZH2 MT and EZH2 WT groups.

Results: As of February 1, 2019, interim data were summarized from $95 \mathrm{FL}$ pts (41 EZH2 MT and 54 EZH2 WT). The overall median age was 61 years, and median prior lines of therapy was 2 (range: 0-11) in the EZH2 MT group and 3 (range: 1-8) in the EZH2 WT group. Median follow-up times, ORR, median duration of response for 92 response evaluable patients in both EZH2 MT and WT groups are highlighted in the table. Response duration was greater than 24 weeks in $83 \%$ of pts, and $50 \%$ of pts had a response duration greater than 1 year. Treatment-emergent adverse events (TEAEs) leading to dose reductions occurred in $8 \%$ of pts and study drug discontinuation due to TEAEs occurred in $9 \%$ of FL pts. The overall safety profile was similar to what has been previously reported. Grade $\geq 3$ treatment-related TEAEs were reported in $18 \%$ of pts; no Grade $5 \mathrm{AEs}$ were reported.

Conclusion: Tazemetostat $800 \mathrm{mg}$ BID appears to be generally well tolerated with observed meaningful clinical activity and durability of response in pts with R/R FL. ORR was pronounced in pts with EZH2 activating mutations. Late onset responses have been reported on tazemetostat. Given the consistently favorable safety and low rates of discontinuation due to TEAEs, these encouraging phase 2 data demonstrate that $\mathrm{EZH} 2$ inhibition may be an important and effective therapeutic target in FL.

Keywords: follicular lymphoma ( $F L$ ); germinal center B cell-like (GCB). Disclosures: Morschhauser, F: Consultant Advisory Role: Gilead, Servier, Roche/Genentech; Honoraria: Celgene, BMS, Janssen. Tilly, H: Consultant Advisory Role: Celgene, Astra-Zeneca, Karyopharm, Roche; Honoraria: BMS, Janssen, Gilead; Research Funding: Celgene. Phillips, T: Consultant Advisory Role: Genentech, Gilead, Bayer, Seattle Genetics, Pharmacyclics; Research Funding: Pharmacyclics, Abbvie. Ribrag, V: Consultant Advisory Role: Epizyme, Servier, Nanostring, Gilead, Pharmamar, BMS, MSD, Incyte, Roche, Infinity; Honoraria: ESAl; Research Funding: Epizyme, ArgenX. Jurczak, W: Consultant Advisory Role: AstraZeneca/Acerta, European Medicines Agency, Sandoz-Nowartis, Janssen, Gilead; Research Funding: Afimed, BeiGene, Celgene, Epizyme, AstraZeneca/Acerta. McKay, P: Consultant Advisory Role: Epizyme; Honoraria: Epizyme. Opat, S: Consultant Advisory Role: Roche, Celgene, Mundipharma, Janssen; Honoraria: Roche, Celgene, Mundipharma, Janssen. Radford, J: Consultant Advisory Role: Takeda, Bristol-Myers Squibb, Seattle Genetics, Novartis; Stock Ownership: GSK, AstraZeneca; Honoraria: Takeda; Research Funding: Takeda, Pfizer, ADC Therapeutics, Celgene, AstraZeneca. Rajarethinam, A: Employment Leadership Position: Epizyme, Inc; Stock Ownership: Epizyme, Inc. Yang, J: Employment Leadership Position: Epizyme, Inc; Stock Ownership: Epizyme, Inc. Howell, H: Employment Leadership Position: Epizyme, Inc; Stock Ownership: Epizyme, Inc. Newberry, K: Employment Leadership Position: Epizyme, Inc; Stock Ownership: Epizyme,

\begin{tabular}{|c|c|c|}
\hline & $\begin{array}{l}\text { EZH2 MT group } \\
\text { ( } n=39 \text { evaluable) }\end{array}$ & $\begin{array}{l}\text { EZH2 WT group } \\
\text { ( } n=53 \text { evaluable) }\end{array}$ \\
\hline Objective response rate, $\%(95 \% \mathrm{CI})$ & $74(57.9,87.0)$ & $34(21.5,48.3)$ \\
\hline Complete response, $\%$ & 10 & 6 \\
\hline Partial response, $\%$ & 64 & 28 \\
\hline Stable disease, \% & 26 & 30 \\
\hline Progressive disease, $\%$ & 0 & 28 \\
\hline Progression-free survival, weeks ( $95 \% \mathrm{Cl}$ ) & $60.0(46.7,83.9)$ & $24.6(15.1,47.9)$ \\
\hline Median duration of response, weeks ( $95 \% \mathrm{Cl}$ ) & $40(22.3, \mathrm{NE})$ & $56(31.7, \mathrm{NE})$ \\
\hline Median follow-up, weeks (min, max) & 64 (5-161 weeks) & 107 (1-178 weeks) \\
\hline
\end{tabular}


Inc. Adib, D: Employment Leadership Position: Epizyme, Inc; Stock Ownership: Epizyme, Inc. Salles, G: Honoraria: Roche, Jannsen, Gilead, Celgene, Novartis, Amgen, BMS, Merck, Servier.

\section{SESSION 9 - EXTRANODAL LYMPHOMAS}

\section{6}

INTEGRATIVE GENOMIC ANALYSIS IDENTIFIES KEY PATHOGENIC CONCEPTS IN PRIMARY MEDIASTINAL LARGE B-CELL LYMPHOMA

\author{
A. Mottok $^{1}$ | S.S. Hung ${ }^{2}$ | E.A. Chavez ${ }^{2}$ | \\ B. Woolcock $^{2}$ | A. Telenius ${ }^{2}$ | L.C. Chong ${ }^{2}$ |

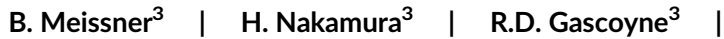

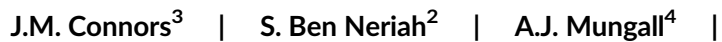 \\ M.A. Marra ${ }^{4}$ ～R.Siebert ${ }^{1}$ | D.W. Scott ${ }^{3}$ | \\ K.J. Savage ${ }^{3}$ | C. Steidl ${ }^{2}$ \\ ${ }^{1}$ Institute of Human Genetics, Ulm University and University Medical \\ Centre, Ulm, Germany; ${ }^{2}$ Department of Lymphoid Cancer Research, BC \\ Cancer, Vancouver, Canada; ${ }^{3}$ Centre for Lymphoid Cancer, BC Cancer, \\ Vancouver, Canada; ${ }^{4}$ Michael Smith Genome Sciences Centre, BC Cancer, \\ Vancouver, Canada
}

Introduction: Primary mediastinal large B-cell lymphoma (PMBCL) is recognized as a distinct entity in the current WHO classification, accounting for $2-3 \%$ of non-Hodgkin lymphomas. Molecular studies have provided evidence that PMBCL can be distinguished from diffuse large B-cell lymphoma (DLBCL) and highlighted a strong relationship between PMBCL and classical Hodgkin lymphoma ( $\mathrm{CHL}$ ). There is cumulative evidence to support that correct classification is important for patient risk stratification and therapeutic decision making. We aimed to comprehensively describe genetic alterations and perturbed pathways in molecularly defined $\mathrm{PMBCL}$, as targeted sequencing studies in the past have focused on a limited number of genetic lesions involved in disease pathogenesis.

Methods: We performed whole-exome sequencing of 95 centrally reviewed $\mathrm{PMBCL}$ cases, using the SureSelect Human All Exon V6 + UTR baits (Agilent) followed by massively parallel sequencing. For 21 cases paired germline DNA was available. Somatic SNV/indel variants were identified using the intersection of predictions by VarScan, Strelka and MuTect, and annotated with SnpEff. We inform on oncogenic driver genes as identified by MutSigCV and recurrent copy number alterations utilizing CNVkit and GISTIC. For 69 patients global gene expression profiling performed on the DASL platform (Illumina) was available and 90 cases have been molecularly classified using the Lymph3Cx assay. The impact of somatic mutations on gene expression was assessed using xseq.
Results: Tumor and normal samples were sequenced to an average coverage of $115 \mathrm{X}$. Analysis of somatic alterations in tumor-normal pairs yielded 50 putative driver genes recurrently mutated in PMBCL. Besides mutations in the JAK-STAT (SOCS1, STAT6, IL4R, PTPN1) and NFkB-pathway (TNFAIP3, NFKBIE, TRAF3), we provide additional evidence of the importance of immune evasion in this disease (CIITA, CD58, B2M) and identified the IRF-pathway as a putative novel hallmark. As expected, the most significant regions of copy number gain predicted by GISTIC were located on $9 p(72 \%)$. The most frequent regions of loss were mapped to $10 q, 8 p, 1 p$, and 7p. PMBCL driver genes, with the exception of EZH2, were significantly more frequently mutated in comparison to DLBCL, whereas only three genes were significantly different between $\mathrm{PMBCL}$ and $\mathrm{CHL}$.

Conclusions: Here we identified candidate driver genes with clear evidence of somatic mutations in $\mathrm{PMBCL}$, the majority being distinct from DLBCL. We observed an enrichment of somatic mutations affecting genes involved in the JAK-STAT- and NFkB-pathway, and propose that the IRF-pathway is critically involved in PMBCL pathogenesis.

Keywords: molecular genetics; primary mediastinal large B-cell lymphoma (PMLBCL).

107

OUTCOME OF PRIMARY MEDIASTINAL LARGE B-CELL LYMPHOMA IN THE RITUXIMAB ERA: IMPACT OF A PETGUIDED APPROACH

\author{
A. Hayden $^{1}$ ｜ P. Tonseth ${ }^{2}$ ｜ D. Villa ${ }^{1}$ | A.S. Gerrie ${ }^{1}$ | \\ D.W. Scott ${ }^{1}$ | C.L. Freeman ${ }^{1}$ | G.W. Slack ${ }^{3}$ |

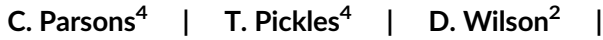 \\ J.M. Connors $^{1}$ ～L.H. Sehn ${ }^{1}$ | K.J. Savage ${ }^{1}$ \\ ${ }^{1}$ Centre for Lymphoid Cancer and Division of Medical Oncology, BC \\ Cancer, Vancouver, Canada; ${ }^{2}$ Division of Radiology, BC Cancer, \\ Vancouver, Canada; ${ }^{3}$ Centre for Lymphoid Cancer and Division of \\ Pathology, BC Cancer, Vancouver, Canada; ${ }^{4}$ Division of Radiation \\ Oncology, BC Cancer, Vancouver, Canada
}

Background: Primary mediastinal large B-cell lymphoma (PMBCL) is a rare subtype of lymphoma which typically presents in females with a bulky anterior mediastinal mass. Patients (pts) are treated with chemoimmunotherapy \pm consolidative radiotherapy (CRT). Herein, we reviewed outcomes of $\mathrm{PMBCL}$ pts treated at $\mathrm{BC}$ Cancer in the rituximab era and the impact of a PET-adapted approach.

Methods: The BC Cancer Lymphoid Cancer Database was used to identify pts $>16$ years (y) of age with newly diagnosed PMBCL based on the WHO classification who were treated with chemotherapy + rituximab. Prior to the availability of FDG-PET scan at end of treatment (EOT) in July 2005, all pts were recommended to receive R- 